\title{
Cancer cell metabolism: implications for therapeutic targets
}

\begin{abstract}
Miran Jang ${ }^{1}$, Sung Soo Kim ${ }^{1}$ and Jinhwa Lee $^{2}$
Cancer cell metabolism is characterized by an enhanced uptake and utilization of glucose, a phenomenon known as the Warburg effect. The persistent activation of aerobic glycolysis in cancer cells can be linked to activation of oncogenes or loss of tumor suppressors, thereby fundamentally advancing cancer progression. In this respect, inhibition of glycolytic capacity may contribute to an anticancer effect on malignant cells. Understanding the mechanisms of aerobic glycolysis may present a new basis for cancer treatment. Accordingly, interrupting lactate fermentation and/or other cancer-promoting metabolic sites may provide a promising strategy to halt tumor development. In this review, we will discuss dysregulated and reprogrammed cancer metabolism followed by clinical relevance of the metabolic enzymes, such as hexokinase, phosphofructokinase, pyruvate kinase M2, lactate dehydrogenase, pyruvate dehydrogenase kinase and glutaminase. The proper intervention of these metabolic sites may provide a therapeutic advantage that can help overcome resistance to chemotherapy or radiotherapy.

Experimental \& Molecular Medicine (2013) 45, e45; doi:10.1038/emm.2013.85; published online 4 October 2013
\end{abstract}

Keywords: aerobic glycolysis; cancer; metabolism; Warburg effect

\section{INTRODUCTION}

Cancer cell metabolism is a direct result of the modulation of intracellular signaling pathways that are disrupted by mutated oncogenes and tumor-suppressor genes. Mutated oncogenic genes can directly initiate cancer cell metabolism. Similarly, mutated metabolic enzymes can facilitate malignant transformation. Metabolism comprises an energy-producing process of which cells have advantages for the maintenance of cell homeostasis as well as growth and proliferation. Normal cells are equipped with complex signaling networks that are orchestrated by key control enzymes, sensing environmental cues and operating metabolic machineries to provide sufficient energy for survival in a perfectly controlled manner. During proliferation, normal cells activate metabolic pathways to accommodate increased adenosine triphosphate (ATP) consumption for cell reproduction. Along with this metabolic boost, unavoidable by-products of aerobic metabolism, such as reactive oxygen species, may unfortunately damage cells and promote DNA mutations. Thus, alterations in cell metabolism may trigger tumorigenesis. Mutations of oncogenes and tumor-suppressor genes can modify multiple intracellular signaling pathways and, in turn, alter cell metabolism to facilitate the tumorigenic process. ${ }^{1}$ Altered signaling pathways not only enable cells to adapt to tumor cell metabolism, but several of these metabolic alterations are also essential for malignant transformation. ${ }^{2}$

The characteristic metabolic hallmark of tumor metabolism is aerobic glycolysis or the Warburg effect; named after Otto Warburg who first described it in 1926. Unlike normal cells that produce energy mostly through the oxidation of pyruvate in the mitochondria, cancer cells predominantly produce energy via enhanced glycolysis in the cytosol, even under aerobic conditions. ${ }^{3}$ Most cancer cells use glycolysis as a means of energy production regardless of whether they are under normoxic or hypoxic condition. ${ }^{4}$ The correlation between glycolytic ATP production and tumor malignancy has

${ }^{1}$ Department of Biochemistry and Molecular Biology, Medical Science and Engineering Research Center for Bioreaction to Reactive Oxygen Species (BK21) and Biomedical Science Institute, School of Medicine, Kyung Hee University, Seoul, Republic of Korea and ${ }^{2}$ Department of Biomedical Laboratory Science, Dongseo University, Busan, Republic of Korea

Correspondence: Dr SS Kim, Department of Biochemistry and Molecular Biology, Medical Science and Engineering Research Center for Bioreaction to Reactive Oxygen Species (BK-21) and Biomedical Science Institute, School of Medicine, Kyung Hee University, \#1, Hoegi-dong, Dongdaemoon-gu, Seoul 130-701, Republic of Korea.

E-mail: sgskim@khu.ac.kr

or Professor J Lee, Department of Clinical Lab Science, School of Health Science, Dongseo University, San 69-1, Jurea-dong, Sasang-gu, Busan, Republic of Korea.

E-mail: jinhwa2000@gdsu.dongseo.ac.kr

Received 21 January 2013; revised 4 July 2013; accepted 10 July 2013 
repeatedly been reported. ${ }^{5}$ It was originally hypothesized that these metabolic changes reflect damage to mitochondrial oxidative phosphorylation, implying that cancer cells could not respire properly to obtain sufficient ATP. However, recent studies revealed that many cancer cells are capable of synthesizing ATP through mitochondrial respiration. ${ }^{6}$ Regardless of whether mitochondrial respiration is reduced, the fact remains that cancer cells exhibit high rates of glycolysis and lactate fermentation, and this dependence on glucose utilization may be exploited for therapeutic intervention.

During the past decade, many studies focusing on mitochondrial function in cancer cells have suggested that the Warburg effect is more closely related to alterations in signaling pathways that control the uptake and utilization of glucose than to mitochondrial defects. The Warburg effect has recently gained attention again in the cancer research field, because researchers have begun to re-evaluate the significance of aerobic glycolysis in tumor cells. ${ }^{7,8}$ Aerobic glycolysis in cancers is the combined result of oncogenes, tumor suppressors, a hypoxic microenvironment, mtDNA mutations and others. Understanding the complex cancer energy metabolism will help to develop new approaches in early diagnosis and cancer therapy. This review will discuss the metabolism of tumor cells, focusing on the analysis of altered enzymatic activities that are involved in aerobic glycolysis, and discuss current strategies of targeting metabolic pathways for cancer treatment.

\section{THE WARBURG EFFECT: METABOLIC REPROGRAMMING}

Glucose is the primary source of energy and is the main fuel for cellular respiration. In glucose utilization in normal conditions, $70 \%$ of ATP is known to be synthesized by oxidative phosphorylation and the rest by glycolysis. As the ATP yield varies with cellular conditions, the ratio between glycolysis and oxidative phosphorylation also varies in different cells, growth states and microenvironments. In hypoxia, for example, enhanced glycolysis compensates the compromised oxidative phosphorylation to maintain the cellular energetic balance. In most solid tumor cells, a switch in metabolism towards glycolysis over respiration in spite of their functional oxidative phosphorylation machinery manifests the cancer-specific aerobic glycolysis. When the oxidative phosphorylation machinery is limited for any reason (hypoxia, suppression of mitochondrial respiration and so on), other pathways such as lactate fermentation are recruited to supply the cellular energy demand. Cancer cells require a vast amount of energy in a short period of time in order to proliferate. Muscle cells in hypoxic conditions and embryo cells during development can adapt to an altered metabolism when oxygen supply is limited or when growth rate exceeds the regular energy supply, respectively. These cells convert glucose to lactate for fast, but less efficient, ATP generation, accompanying high glucose consumption. Likewise, cancer cells exhibit altered metabolism to meet energy needs during tumor progression; which is known as the Warburg effect.

The Warburg effect involves a metabolic shift from oxidative phosphorylation to glycolysis or lactate fermentation, even in the presence of oxygen. ${ }^{3}$ Otto Warburg originally proposed that a mitochondrial defect disrupted mitochondrial respiration resulting in an increase in glycolysis to meet the energy demand of cancer cells. Alternatively, hypoxia, which is common in cancerous tumors, can relieve oxygen inhibition of the glycolytic pathway (Pasteur's effect), resulting in anaerobic glycolysis. However, it was later determined that cancer cells utilize aerobic glycolysis for energy generation instead of mitochondrial respiration, even in the presence of normally functioning mitochondria. Converting glucose to lactate, rather than metabolizing it through mitochondrial oxidative phosphorylation, is far less efficient, as the former process generates fewer ATP molecules per unit of glucose. Therefore, a high rate of glucose uptake is required to meet the increased energy needs involved in supporting rapid tumor progression. Collectively, these processes all contribute to the Warburg effect. The dependency on fermentative metabolism for ATP generation is a long-term metabolic reprogramming in transformed cancer cells where glucose uptake and glycolytic activities are markedly increased. Even under normoxia, an acute repressive signaling cascade imposed onto mitochondrial function is commonly observed. Transformation of normal cells into cancer cells involves a long-lasting irreversible metabolic switch toward activation of glucose transport and utilization in addition to the suppression of mitochondrial respiration. ${ }^{4}$ If the function of respiratory machinery is normal, the glycolytic activity will be regulated via different pathways to maintain a balance of energy. ${ }^{9}$ Although glutamine was not emphasized by Warburg, glutamine is an essential bioenergetic and anabolic substrate for many cancer cell types. Cancer cells exhibiting aerobic glycolysis rely on glutamine as well as glucose as the carbon source. Glutamine is used to provide intermediates of the tricarboxylic acid (TCA) cycle to feed other biosynthetic pathways as precursors. Therefore, cancer cells are dependent on glutamine to maintain the TCA cycle, as they rely on glucose during aerobic glycolysis.

Many studies have shown that metabolic alterations of glucose provide additional energy to support tumor growth. Therefore, the inhibition of glycolysis results in reduced cell proliferation and tumor growth. Moreover, the inhibition of biosynthetic pathways that are related to glycolysis decreases tumor growth. These suggest that targeting glycolysis may be a useful strategy for preventing or stopping the development of cancer.

\section{ONCOGENIC SIGNALS AND METABOLIC REPROGRAMMING}

Mutations that activate oncogenes or inactivate tumor suppressors can significantly affect activities of metabolic enzymes and have a key role in aerobic glycolysis of cancer. ${ }^{10-12}$ Among oncogenic mutations, alterations in phosphatidylinositol $3^{\prime}$-kinase (PI3K), phosphatase and tensin homolog (PTEN), Myc and p53 can all impact cellular metabolism (Figure 1). The PI3K pathway has frequently been shown to be altered, and thus has a major role in tumor proliferation and survival for a wide variety of human cancers. ${ }^{13}$ Activation of PI3K 


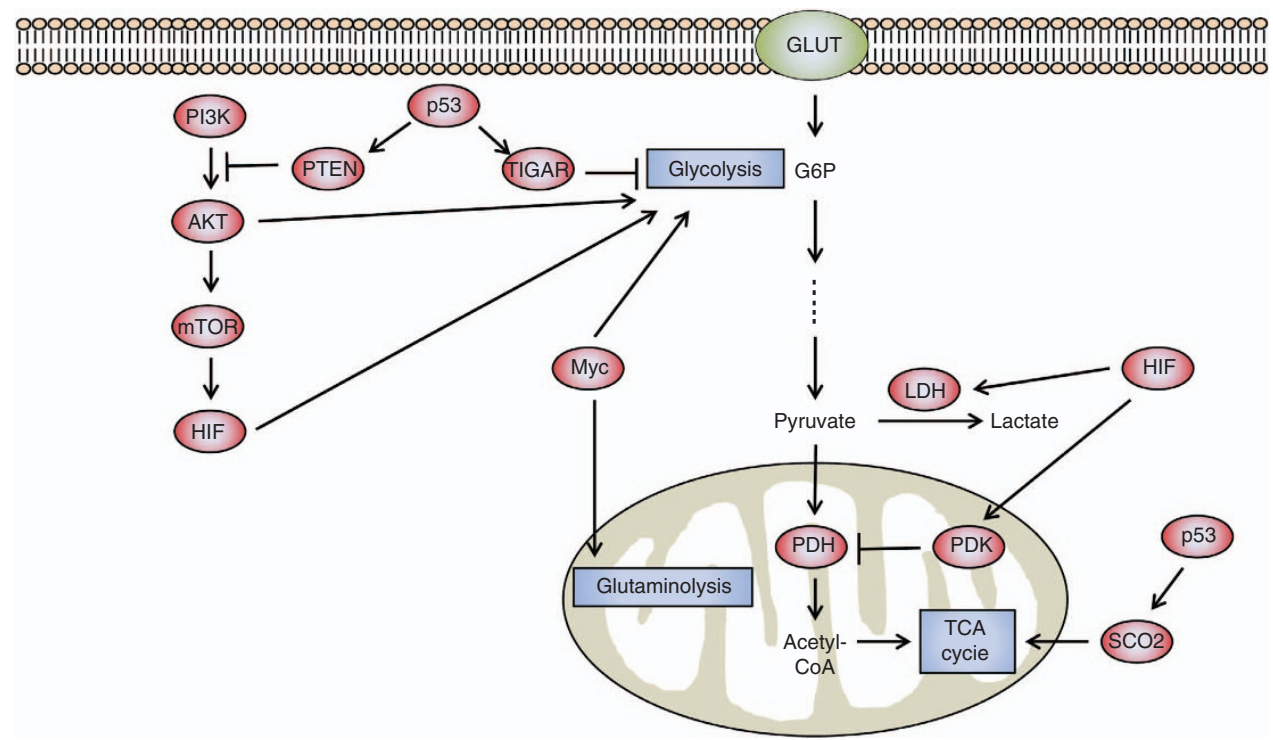

Figure 1 Metabolism of cancer cells is regulated by signaling pathways related to oncogenes and tumor-suppressor genes. PI3K activates AKT, which stimulates glucose uptake and flux by directly controlling glycolytic enzymes and by activating mTOR. mTOR indirectly causes metabolic changes by activating HIF. HIF activates PDK, which inactivates the mitochondrial pyruvate dehydrogenase complex and thereby inhibits the entry of pyruvate into the TCA cycle. p53 suppresses glycolysis by increasing the expression of TIGAR, supporting the expression of PTEN, and promoting oxidative phosphorylation via SCO2. Myc enhances the glycolytic pathway by increasing transcription of glycolytic enzymes and is also involved in glutamine metabolism.

results in the downstream activation of AKT and stabilization of hypoxia-inducible factor (HIF)-1. The PI3K enzyme itself antagonizes the tumor-suppressor PTEN, and the loss of PTEN increases glycolysis by activation of AKT and HIF-1. ${ }^{10}$ AKT stimulates glycolysis by increasing the expression and membrane translocation of glucose transporters, and also by phosphorylation of glycolytic enzymes, such as hexokinase (HK) and phosphofructokinase (PFK). ${ }^{10,14}$ Moreover, AKT activates mammalian target of rapamycin (mTOR), which indirectly affects other metabolic pathways by activating HIF1 , even under normoxic conditions. ${ }^{14}$ Oncogene mutation of Ras activates mTOR via the PI3K-Akt-mTOR signaling pathway, and mTOR promotes glycolysis through inducing HIFs. ${ }^{15-19}$ The HIFs are transcription factors that regulate gene expression in response to the cellular environment, specifically in the event of decreased oxygen or hypoxia. HIF-1 regulates many different genes such as vascular endothelial growth factor, hepatocyte growth factor receptor (c-Met), erythropoietin, transforming growth factor- $\alpha$, platelet-derived growth factor$\beta$ and glucose transporter GLUT1 and therefore influences such cellular activities as angiogenesis, glycolysis and cell survival. ${ }^{20}$ Also, HIF-1 activates pyruvate dehydrogenase kinases (PDKs), thereby inactivating pyruvate dehydrogenase and blocking the flow of pyruvate into the TCA cycle. ${ }^{21-23}$ Reduced transport of pyruvate into the mitochondria decreases the rate of oxidative phosphorylation and oxygen consumption.

The oncogenic transcription factor, Myc, also directly activates transcription of glycolytic enzymes that affect cell metabolism. Myc was shown to regulate glycolysis in cells grown under normoxic conditions through the direct activation of lactate dehydrogenase A and virtually all other glyco- lytic genes. ${ }^{24}$ Myc can also activate genes associated with mitochondrial biogenesis and function and cooperates with HIF to activate certain glucose transporters and glycolytic enzymes, as well as lactate dehydrogenase A and PDK $1 .{ }^{25,26}$ In contrast to AKT and HIF, Myc can regulate genes associated with glutamine metabolism. ${ }^{27-29}$ Collectively, these alterations induce high levels of glycolysis and glutaminolysis in Mycinduced liver cancer, which are associated with an aggressive tumor phenotype and histology. ${ }^{30}$ It is important to note that even though Myc can enhance the glycolytic pathway, it also enhances mitochondrial respiration, all of which contribute to the overall increased metabolic function of cancer cells.

The tumor-suppressor p53 is probably one of the single most important proteins mutated in many human cancers. In addition to its role in cell cycle control and cell death, p53 also shows an inhibitory effect on glycolysis. $^{31}$ p53 upregulates the expression of TP53-induced glycolysis and apoptosis regulator (TIGAR), which decreases fructose-2,6bisphosphatase (Fru-2,6- $\mathrm{P}_{2}$ ) by dephosphorylation. Increased expression of TIGAR results in a decreased level of Fru-2,6- $\mathrm{P}_{2}$ and a decreased glycolytic rate. ${ }^{32}$ Furthermore, p53 directly stimulates oxidative phosphorylation through upregulation of cytochrome $c$ oxidase 2 (SCO2), which is required for the assembly of the cytochrome $c$ oxidase complex of the electron transport chain. ${ }^{33}$ Hence, loss of p53 shifts metabolism from mitochondrial respiration towards glycolysis.

\section{MUTATIONS OF METABOLIC GENES IN CANCER}

Although the Warburg effect describes altered cancer metabolism, mutations in genes that encode metabolic enzymes can provide a direct genetic link to the altered metabolism 


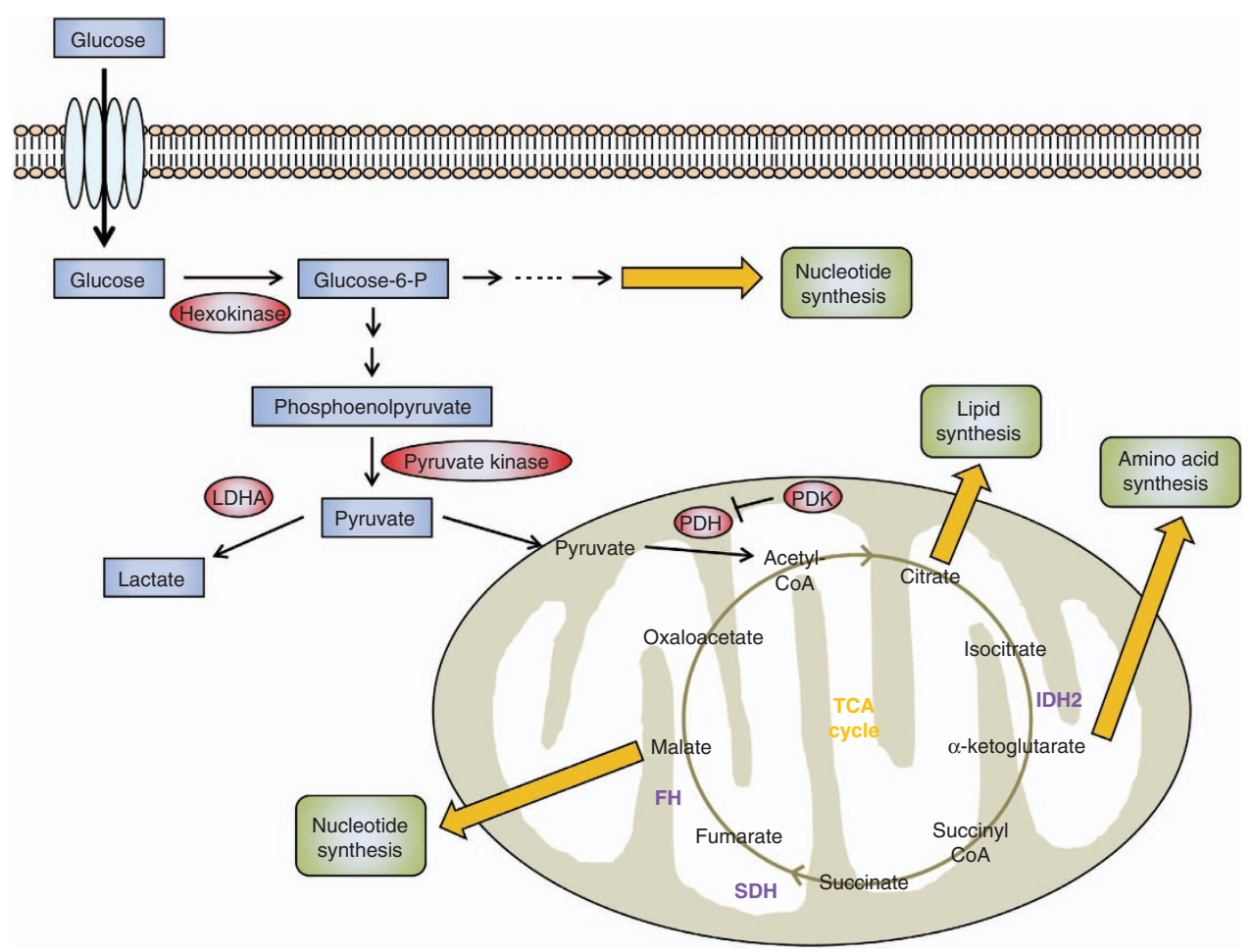

Figure 2 The regulation of glucose metabolism in cancer cells. When glucose enters the cell through a glucose transporter, it is phosphorylated by HK to glucose-6-phosphate, which is further metabolized by glycolysis to pyruvate in the cytosol. Under aerobic conditions, normal cells use pyruvate dehydrogenase (PDH) to convert most pyruvate to acetyl-CoA. The acetyl-CoA is then oxidized via the TCA cycle, providing sources of ATP synthesis. In contrast, the metabolic pathways of glucose utilization in cancer are changed from ATP generation by oxidative phosphorylation to ATP generation through glycolysis. Also, for cell proliferation to occur, cancer cells require the synthesis of new macromolecules (for example, nucleic acids, lipids, proteins). Key enzymes that may be promising targets for cancer therapy are highlighted in red. TCA enzymes that are known to be mutated in cancer are shown in purple: IDH2, SDH, and FH.

(Figure 2). Mutations in two TCA cycle-related genes that encode fumarate hydratase (FH) and succinate dehydrogenase $(\mathrm{SDH})$ lead to accumulation of their respective substrates, fumarate and succinate, in a subset of human cancers. SDH also serves as respiratory complex II in the electron transport chain; therefore mutations in SDH may directly affect mitochondrial respiration. Indeed, mutations in the genes encoding SDH subunits have been connected to hereditary parangangliomas and pheochromocytomas. ${ }^{34-36}$ Additionally, $\mathrm{FH}$ gene encodes the enzyme that converts fumarate to malate in the mitochondria. Thus, mutations in FH result in fumarate hydratase deficiency and have also been connected to uterine and skin leiomyomas and papillary renal cancer. ${ }^{37}$

Connections between a mutant metabolic enzyme and tumorigenesis have been uncovered from cancer genomesequencing efforts. NADP (nicotinamide adenine dinucleotide phosphate)-dependent isocitrate dehydrogenase 1 (IDH1) and IDH2 provide a fascinating example of a mutant metabolic enzyme driving tumorigenesis. IDH1 and IDH2 catalyze the conversion of isocitrate to $\alpha$-ketoglutarate $(\alpha \mathrm{KG})$ in mammalian cells with the generation of one molecule of NADPH. IDH1 and IDH2 are homodimeric enzymes that act in the cytoplasm and mitochondria, respectively. It has been shown that heterozygous point mutations in several residues in IDH1 are common in gliomas and in acute myeloid leukemia. ${ }^{38,39}$ These mutations have been shown to act in a dominantnegative fashion to inhibit IDH1 and IDH2 activity, leading to a reduction in cytoplasmic $\alpha$ KG concentration, an inhibition of prolyl hydroxylase activity and a stabilization of the hypoxia-induced transcription factor HIF- $1{ }^{40}$ Additionally, these mutations have been reported to provide IDH1 and IDH2 with novel enzymatic activity, causing conversion of $\alpha \mathrm{KG}$ to 2-hydroxyglutarate (2-HG), ${ }^{41-43}$ the effects of which are not well understood. Although 2-HG is present at low concentrations in normal cells and tissues, mutations in IDH1 and IDH2 result in elevated 2-HG levels in glioma tissues and in acute myeloid leukemia cells. ${ }^{41-43}$ Hence, it would be interesting to know if high concentrations of $2-\mathrm{HG}$ are directly related to the tumorigenicity of IDH1 and IDH2 mutations. Importantly, levels of $\alpha \mathrm{KG}$, isocitrate and several other TCA metabolites remain unchanged in cells or tissues with IDH1 mutations, suggesting that other metabolic pathways can adjust and maintain normal levels of essential metabolites. ${ }^{41,43}$

A common feature associated with mutations in the metabolic enzymes SDH, FH and IDH is an aberrant accumulation of metabolites with oncogenic potential. The metabolic products of mutant SDH, FH and IDH inhibit the activity of 
enzymes (for example, prolyl hydroxylases) belonging to a class of $\alpha$ KG-dependent enzymes. Interestingly, these $\alpha$ KG-dependent enzymes normally target HIF for degradation. Furthermore, mutant SDH, FH and IDH have all been shown to induce HIF activity. ${ }^{44}$ Thus, oncogenic alterations in cellular metabolism can regulate pathways that are unrelated to metabolism and that contribute to the oncogenic process, such as altered HIF activity.

Altered mitochondrial metabolism can have an important role in carcinogenesis. Phosphoglycerate dehydrogenase (PHGDH) and glycine decarboxylase are two enzymes showing a similar pattern of mutation that can both affect mitochondrial metabolism directly during oncogenesis. PHGDH catalyzes the first step in serine synthesis, ${ }^{45,46}$ is amplified in estrogen receptornegative breast cancers, and is suggested to be an oncogenic enzyme when overexpressed. PHGDH also shuttles glycolytic intermediates into the one-carbon metabolic pathway and thus directs nucleotide biosynthesis. Loss of PHGDH decreases the level of a key TCA intermediate, $\alpha \mathrm{KG}$, but surprisingly not serine. ${ }^{46}$ Glycine decarboxylase is also involved in glycine/serine metabolism and the one-carbon metabolic pathway, and its overexpression promotes tumorigenesis. ${ }^{47}$

\section{METABOLIC TARGETING}

Metabolic targeting for cancer therapy is currently under investigation in an effort to identify small molecules that might specifically inhibit key metabolic steps associated with tumor growth (Table 1). Attenuation or inhibition of glycolysis has been found useful for preventing the development of cancer, demonstrating that glycolysis is essential for proliferation, invasion and metastasis of cancer. ${ }^{48-50}$ Glycolysis can be blocked by inhibition of the glycolytic enzymes HK, PFK and pyruvate kinase $(\mathrm{PK})$, all of which regulate irreversible and rate-limiting steps in glycolysis. Therefore, the strategy is to compromise or completely block the increased glycolysis in cancer cells by abating the enzymatic activity of these three proteins.

HK facilitates the first step of glycolysis, where glucose is phosphorylated with the use of ATP. Inhibitors of HK such as 2-deoxyglucose (2-DG), 3-bromopyruvate (3-BrPA) and lonidamine (LON, 1-[(2,4-dichlorophenyl)methyl]-1H-indazole3 -carboxylic acid) are currently in pre-clinical and early phase clinical trials. 2-DG serves as a competitive inhibitor of HK, blocking access of glucose to the enzyme. 2-DG is taken up by glucose transporters and phosphorylated by HK to 2-DG-P and thereby trapped within the cell, because 2-DG-P is not recognized and metabolized by the next glycolytic enzyme, phosphoglucose isomerase. This leads to the accumulation of 2-DG-P within the cell and a depletion of cellular ATP production. ${ }^{51}$ However, its effectiveness as a single agent is limited and combining 2-DG with radiotherapy or chemotherapy enhances the tumor-destroying effects. ${ }^{52} 3$ - $\mathrm{BrPA}$ is known to inhibit cancer cell energy metabolism and is a key determinant of chemoresistance in certain cancer types. $^{53,54}$ ATP depletion caused by glycolysis inhibition with 3-BrPA treatment suppresses ATP-binding cassette (ABC) transporter activity and subsequently drug efflux, enhancing drug retention. As a result, 3-BrPA can improve cancer therapeutics or overcome chemoresistance. Consistently, it was previously reported that increased glycolysis is directly associated with glucocorticoid resistance that is associated with treatment failure in childhood acute lymphoblastic leukemia and inhibition of glycolysis by 2-DG, 3-BrPA or LON increases prednisolone-induced toxicity in leukemia cells. ${ }^{55}$ LON, 1-[(2,4-dichlorophenyl)methyl]-1H-indazole-3-carboxylic acid, is a HK 2 inhibitor that has been shown to induce apoptosis and treat multidrug resistance in various cancer cell lines. ${ }^{56-58}$ However, Phase II clinical trials of LON as a treatment for benign prostatic hyperplasia have been suspended because of its liver toxicity. ${ }^{59,60}$ The combination of LON with other anticancer drugs like Paclitaxel, a common chemotherapeutic agent that hyper-stabilizes microtubules and prevents cell division but shows high toxicity, is undergoing clinical trials for the treatment of cancers. ${ }^{51,61}$

PFK activity is extremely sensitive to small changes in $\mathrm{pH}$, and its activity drops as $\mathrm{pH}$ decreases. ${ }^{62-64}$ The intracellular $\mathrm{pH}$ in cancer cells is typically high, activates the rate-limiting enzyme PFK and subsequently facilitates glycolysis. ${ }^{65-67} \mathrm{The} \mathrm{Na}^{+} / \mathrm{H}^{+}$exchanger NHE-1 is known to increase the intracellular $\mathrm{pH}$ in cancer cells. It has been reported that inhibitors of NHE-1 such as amiloride or 5,5-dimethylamiloride produce anticancer effects by decreasing intracellular $\mathrm{pH}$, PFK activity and thus decreasing glycolysis. ${ }^{65,68,69}$ Citrate is another potential inhibitor of PFK activity. Citrate is an archetypal feedback inhibitor of PFK and is normally converted into cytosolic acetyl-CoA by the enzyme ATP citrate lyase. However, use of an ATP citrate lyase inhibitor,

Table 1 Compounds targeting cancer metabolism

\begin{tabular}{llll}
\hline Metabolism & Compound & Target & Action \\
\hline Glycolysis & Phloretin & GLUT1 & Inhibits glucose uptake \\
Glycolysis & WZB117 & GLUT1 & Inhibits glucose uptake \\
Glycolysis & 2-Deoxyglucose & Hexokinase & Inhibits glycolytic flux \\
Glycolysis & 3-Bromopyruvate & Hexokinase & Inhibits glycolytic flux \\
Glycolysis & Lonidamine & Hexokinase & Inhibits glycolytic flux \\
Glycolysis & FX11 & Lactate dehydrogenase & Inhibits pyruvate metabolism \\
Glycolysis & Oxamate & Lactate dehydrogenase & Inhibits pyruvate metabolism \\
Tricarboxylic acid cycle & Dichloroacetate & Pyruvate dehydrogenase kinase & Promotes oxidation in the mitochondria \\
Fatty acid synthesis & C75 & FASN & Inhibits fatty acid synthesis
\end{tabular}


SB-204990, can increase citrate levels that, in turn, inhibit glycolysis, ${ }^{70-72}$ thereby producing anticancer effects.

The PK isozyme M2 (PKM2) is present in very few types of normal proliferating cells but is highly expressed in cancer cells. $^{73,74}$ PKM2 catalyzes the rate-limiting ATP-generating step of glycolysis, controlling the conversion of phosphoenolpyruvate to pyruvate. ${ }^{74}$ By inhibiting PKM2, the glycolytic flux regresses to limit the generation of ATP in cancer cells. Although such an effect might seem to be detrimental to tumor growth, the result is actually the opposite. Similar to normal cells, cancer cells must generate macromolecules such as lipids, nucleic acids and proteins. Without the biosynthesis of these macromolecules, rapid cell proliferation will cease even in the presence of ATP. Slowing glycolysis and shuttling of phosphoenolpyruvate through the pentose phosphate pathway generates macromolecule precursors that are necessary to support cell proliferation ${ }^{75-77}$ and hence counteract the effects of inhibited glycolysis. Despite this setback, PKM2 is still of interest, because it is uniquely important to tumor metabolism.

Until recently, the importance of glutamine metabolism and its regulation has not been emphasized for tumor metabolism, because the dependency of cancer cells on glutamine was not fully appreciated by Warburg. Modulation of transamination, the transfer of an amine from glutamine during mitochondrial respiration, has been proposed to inhibit tumor growth. ${ }^{78,79}$ It consists of glutaminase that converts glutamine to glutamate and glutamate dehydrogenase that converts glutamate to $\alpha \mathrm{KG}$. Myc has been shown to regulate many steps in glutamine metabolism, which gives an insight on important enzymes in glutamine metabolism as therapeutic targets when metabolism is reprogrammed in cancer cells. ${ }^{80}$ It has been suggested that modulation of both transamination and PK can be a combined method for metabolic targeting of tumors. Metabolites from transamination may also have a regulatory role on the dimer/tetramer structure of PK. A balanced presence of both PK structures is essential for enzymatic activity, and modulation of the dimer/tetramer ratio by transamination metabolites can be detrimental for tumor growth.

Lactate dehydrogenase (LDH) is another enzyme important for tumor cell metabolism. $\mathrm{LDH}$ catalyzes the conversion of pyruvate to lactate. This reduction step is absolutely necessary for the regeneration of $\mathrm{NAD}^{+}$, which is needed to continue glycolysis. It has been observed that decreases in $\mathrm{LDH}$ inhibits glycolysis and has antitumor effects in cancer cells. ${ }^{81,82}$ Deck et al. ${ }^{83}$ have synthesized dihydroxynaphthoic acids, which are potent inhibitors of $\mathrm{LDH}$ and inhibitors of glycolysis. The specific LDH inhibitor, oxamate, may also be useful in treating paclitaxel-resistant cancers. Combinational treatment of paclitaxel and oxamate showed a synergistic inhibitory effect in the paclitaxel-resistant breast cancer cells by promoting apoptosis. ${ }^{84}$ Lastly, inhibition of PDK reduces glycolysis and promotes mitochondrial function by activating pyruvate dehydrogenase, and reduction of glycolysis may lead to an antitumor response. Many studies have shown that dichloroacetate, a PDK inhibitor, produced markedly positive outcomes for the treatment of lactic acidosis and mitochondrial diseases ${ }^{85,86}$ and may prove useful in the treatment of cancer.

\section{PERSPECTIVES}

Cancer cell metabolism utilizes aerobic glycolysis in which cancer cells use glucose for energy supply and glutamine to feed mitochondrial intermediates for biosynthetic precursor supply. Altered metabolism is considered to be fundamental to the transformation of normal cells to cancer cells, and it is believed to be conserved in most tumors, including solid tumors, lymphoma and leukemia. Certainly, cancers display extremely heterogeneous characteristics as tissue origin in every cancer type is different. ${ }^{87}$ Also, cancer cells suffer from poor nutrient and oxygen supplies due to the imperfect vasculature formed during tumorigenesis. ${ }^{20,88,89}$ It is worth noting that cancer cells exhibit greater plasticity than normal cells. ${ }^{90}$ Therefore, alterations of metabolic pathways in tumor cells may give rise to a selective advantage for rapid generation of ATP and for sufficient biomolecule production to cancer cells under the unfavorable environment. ${ }^{91,92}$ Hypoxic adaptation essential for survival and progression of a tumor is thought to be closely linked to metabolic changes in cancer cells. Along with a reduced use of oxygen and the rapid energy production, there is a notable shift in mitochondrial function from an energy producer to a creator of biosynthetic intermediates. Although the molecular mechanisms still remain largely unknown, mutations of oncogenes and tumor suppressors account for part of the metabolic reprogramming in cancer cells. Cancer cell metabolism has recently become one of the most exciting and promising fields for the development of new anticancer agents. Cancer research encompasses distinct fields of molecular, biological and metabolic research areas, and until recently, only signal transduction pathways have been recognized as a promising field for the development of therapeutic drugs against tumors. By better understanding the cancer-specific metabolic process, and by further comprehending the tumor cell, researchers in the field of metabolic research hope to find new drugs that may revolutionize cancer treatment. Targeting cancer metabolism opens an opportunity to develop broadly applicable drugs that can treat multiple cancer types and hence may lead to a new class of anticancer drugs. Numerous analogs of metabolites are being tested at present as potential drug candidates to target tumor metabolism. Further studies on the role of the mitochondria in cancer cell metabolism may provide a selective delivery system for toxic chemicals specifically to cancer cells, thereby increasing the efficacy and reducing the toxicity of potentially powerful chemotherapeutic drugs. New understandings of cancer metabolic profiles give us a hope that a new class of therapeutic agents may be developed for cancer therapy. Therefore, effective use of metabolic inhibitors may provide clinically favorable therapeutic strategy.

\section{ACKNOWLEDGEMENTS}

This work was supported by the Dongseo University, 'Dongseo Frontier Project' Research Fund of 2010 to JL and by the National 
Research Foundation of Korea (NRF) Grant (No. 2012-0009380) from the Korea Ministry of Education, Science and Technology (MEST) to SSK and NRF Grant (No. 2010-0024592) to JL. This work was conducted during the Sabbatical year by JL in the Dongseo University.

1 Vogelstein B, Kinzler KW. Cancer genes and the pathways they control. Nat Med 2004; 10: 789-799.

2 DeBerardinis RJ, Lum JJ, Hatzivassiliou G, Thompson CB. The biology of cancer: metabolic reprogramming fuels cell growth and proliferation. Cell Metab 2008; 7: 11-20.

3 Warburg O. On the origin of cancer cells. Science 1956; 123: 309-314.

4 Koppenol WH, Bounds PL, Dang CV. Otto Warburg's contributions to current concepts of cancer metabolism. Nat Rev Cancer 2011; 11: 325-337.

5 Simonnet H, Alazard N, Pfeiffer K, Gallou C, Beroud C, Demont J et al. Low mitochondrial respiratory chain content correlates with tumor aggressiveness in renal cell carcinoma. Carcinogenesis 2002; 23: 759-768.

6 Weinhouse S. The Warburg hypothesis fifty years later. $Z$ Krebsforsch Klin Onkol Cancer Res Clin Oncol 1976; 87: 115-126.

7 Dang CV, Hamaker M, Sun P, Le A, Gao P. Therapeutic targeting of cancer cell metabolism. J Mol Med (Berl) 2011; 89: 205-212.

8 Zhao Y, Butler EB, Tan M. Targeting cellular metabolism to improve cancer therapeutics. Cell Death Dis 2013; 4: e532.

9 Pfeiffer T, Schuster S, Bonhoeffer S. Cooperation and competition in the evolution of ATP-producing pathways. Science 2001; 292: 504-507.

10 Elstrom RL, Bauer DE, Buzzai M, Karnauskas R, Harris MH, Plas DR et al. Akt stimulates aerobic glycolysis in cancer cells. Cancer Res 2004; 64: 3892-3899.

11 Levine AJ, Puzio-Kuter AM. The control of the metabolic switch in cancers by oncogenes and tumor suppressor genes. Science 2010; 330: 1340-1344.

12 Bayley JP, Devilee P. The Warburg effect in 2012. Curr Opin Oncol 2012; 24: 62-67.

13 Wong KK, Engelman JA, Cantley LC. Targeting the PI3K signaling pathway in cancer. Curr Opin Genet Dev 2010; 20: 87-90.

14 Robey RB, Hay N. Is Akt the "Warburg kinase"?-Akt-energy metabolism interactions and oncogenesis. Semin Cancer Biol 2009; 19: 25-31.

15 Denko NC. Hypoxia, HIF1 and glucose metabolism in the solid tumour. Nat Rev Cancer 2008; 8: 705-713.

16 Majmundar AJ, Wong WJ, Simon MC. Hypoxia-inducible factors and the response to hypoxic stress. Mol Cell 2010; 40: 294-309.

17 Duvel K, Yecies JL, Menon S, Raman P, Lipovsky Al, Souza AL et al. Activation of a metabolic gene regulatory network downstream of mTOR complex 1. Mol Cell 2010; 39: 171-183.

18 Pylayeva-Gupta Y, Grabocka E, Bar-Sagi D. RAS oncogenes: weaving a tumorigenic web. Nat Rev Cancer 2011; 11: 761-774.

19 Yecies JL, Manning BD. mTOR links oncogenic signaling to tumor cell metabolism. J Mol Med (Berl) 2011; 89: 221-228.

20 Semenza GL. HIF-1: upstream and downstream of cancer metabolism. Curr Opin Genet Dev 2010; 20: 51-56.

21 Kim JW, Tchernyshyov I, Semenza GL, Dang CV. HIF-1-mediated expression of pyruvate dehydrogenase kinase: a metabolic switch required for cellular adaptation to hypoxia. Cell Metab 2006; 3: 177-185.

22 Papandreou I, Cairns RA, Fontana L, Lim AL, Denko NC. HIF-1 mediates adaptation to hypoxia by actively downregulating mitochondrial oxygen consumption. Cell Metab 2006; 3: 187-197.

$23 \mathrm{Lu} \mathrm{CW}$, Lin SC, Chen KF, Lai YY, Tsai SJ. Induction of pyruvate dehydrogenase kinase-3 by hypoxia-inducible factor- 1 promotes metabolic switch and drug resistance. J Biol Chem 2008; 283: 28106-28114.

24 Dang CV, O'Donnell KA, Zeller KI, Nguyen T, Osthus RC, Li F. The c-Myc target gene network. Semin Cancer Biol 2006; 16: 253-264.

25 Kim JW, Gao P, Liu YC, Semenza GL, Dang CV. Hypoxia-inducible factor 1 and dysregulated c-Myc cooperatively induce vascular endothelial growth factor and metabolic switches hexokinase 2 and pyruvate dehydrogenase kinase 1. Mol Cell Biol 2007; 27: 7381-7393.

26 Dang CV, Kim JW, Gao P, Yustein J. The interplay between MYC and HIF in cancer. Nat Rev Cancer 2008; 8: 51-56.

27 Gao P, Tchernyshyov I, Chang TC, Lee YS, Kita K, Ochi T et al. c-Myc suppression of $\mathrm{miR}-23 \mathrm{a} / \mathrm{b}$ enhances mitochondrial glutaminase expression and glutamine metabolism. Nature 2009; 458: 762-765.
28 Li F, Wang Y, Zeller KI, Potter JJ, Wonsey DR, O'Donnell KA et al. Myc stimulates nuclearly encoded mitochondrial genes and mitochondrial biogenesis. Mol Cell Biol 2005; 25: 6225-6234.

29 Wise DR, DeBerardinis RJ, Mancuso A, Sayed N, Zhang XY, Pfeiffer HK et al. Myc regulates a transcriptional program that stimulates mitochondrial glutaminolysis and leads to glutamine addiction. Proc Natl Acad Sci USA 2008; 105: 18782-18787.

30 Hu S, Balakrishnan A, Bok RA, Anderton B, Larson PE, Nelson SJ et al. 13C-pyruvate imaging reveals alterations in glycolysis that precede c-Myc-induced tumor formation and regression. Cell Metab 2011; 14: 131-142.

31 Vousden KH, Ryan KM. p53 and metabolism. Nat Rev Cancer 2009; 9: 691-700.

32 Bensaad K, Tsuruta A, Selak MA, Vidal MN, Nakano K, Bartrons R et al. TIGAR, a p53-inducible regulator of glycolysis and apoptosis. Cell 2006; 126: $107-120$.

33 Matoba S, Kang JG, Patino WD, Wragg A, Boehm M, Gavrilova 0 et al. p53 regulates mitochondrial respiration. Science 2006; 312: 1650-1653.

34 Baysal BE, Ferrell RE, Willett-Brozick JE, Lawrence EC, Myssiorek D, Bosch A et al. Mutations in SDHD, a mitochondrial complex II gene, in hereditary paraganglioma. Science 2000; 287: 848-851.

35 Niemann S, Muller U. Mutations in SDHC cause autosomal dominant paraganglioma, type 3. Nat Genet 2000; 26: 268-270.

36 Astuti D, Latif F, Dallol A, Dahia PL, Douglas F, George E et al. Gene mutations in the succinate dehydrogenase subunit SDHB cause susceptibility to familial pheochromocytoma and to familial paraganglioma. Am J Hum Genet 2001; 69: 49-54.

37 Tomlinson IP, Alam NA, Rowan AJ, Barclay E, Jaeger EE, Kelsell D et al. Germline mutations in $\mathrm{FH}$ predispose to dominantly inherited uterine fibroids, skin leiomyomata and papillary renal cell cancer. Nat Genet 2002; 30: 406-410.

38 Parsons DW, Jones S, Zhang X, Lin JC, Leary RJ, Angenendt P et al. An integrated genomic analysis of human glioblastoma multiforme. Science 2008; 321: 1807-1812.

39 Mardis ER, Ding L, Dooling DJ, Larson DE, McLellan MD, Chen K et al. Recurring mutations found by sequencing an acute myeloid leukemia genome. N Engl J Med 2009; 361: 1058-1066.

40 Zhao S, Lin Y, Xu W, Jiang W, Zha Z, Wang P et al. Glioma-derived mutations in IDH1 dominantly inhibit IDH1 catalytic activity and induce HIF-1alpha. Science 2009; 324: 261-265.

41 Gross S, Cairns RA, Minden MD, Driggers EM, Bittinger MA, Jang HG et al. Cancer-associated metabolite 2-hydroxyglutarate accumulates in acute myelogenous leukemia with isocitrate dehydrogenase 1 and 2 mutations. J Exp Med 2010; 207: 339-344.

42 Ward PS, Patel J, Wise DR, Abdel-Wahab O, Bennett BD, Coller HA et al. The common feature of leukemia-associated IDH1 and IDH2 mutations is a neomorphic enzyme activity converting alpha-ketoglutarate to 2-hydroxyglutarate. Cancer Cell 2010; 17: 225-234.

43 Dang L, White DW, Gross S, Bennett BD, Bittinger MA, Driggers EM et al. Cancer-associated IDH1 mutations produce 2-hydroxyglutarate. Nature 2009; 462: 739-744.

44 Bayley JP, Devilee P. Warburg tumours and the mechanisms of mitochondrial tumour suppressor genes. Barking up the right tree? Curr Opin Genet Dev 2010; 20: 324-329.

45 Locasale JW, Grassian AR, Melman T, Lyssiotis CA, Mattaini KR, Bass AJ et al. Phosphoglycerate dehydrogenase diverts glycolytic flux and contributes to oncogenesis. Nat Genet 2011; 43: 869-874.

46 Possemato R, Marks KM, Shaul YD, Pacold ME, Kim D, Birsoy K et al. Functional genomics reveal that the serine synthesis pathway is essential in breast cancer. Nature 2011; 476: 346-350.

47 Zhang WC, Shyh-Chang N, Yang H, Rai A, Umashankar S, Ma S et al. Glycine decarboxylase activity drives non-small cell lung cancer tumorinitiating cells and tumorigenesis. Cell 2012; 148: 259-272.

48 Lopez-Lazaro M. Does hypoxia really control tumor growth? Cell Oncol 2006; 28: 327-329.

49 Gatenby RA, Gawlinski ET, Gmitro AF, Kaylor B, Gillies RJ. Acid-mediated tumor invasion: a multidisciplinary study. Cancer Res 2006; 66: 5216-5223.

50 Lopez-Lazaro M. Why do tumors metastasize? Cancer Biol Ther 2007; 6: 141-144.

51 Pelicano H, Martin DS, Xu RH, Huang P. Glycolysis inhibition for anticancer treatment. Oncogene 2006; 25: 4633-4646.

52 Maschek G, Savaraj N, Priebe W, Braunschweiger P, Hamilton K, Tidmarsh GF et al. 2-deoxy-D-glucose increases the efficacy of adriamycin 
and paclitaxel in human osteosarcoma and non-small cell lung cancers in vivo. Cancer Res 2004; 64: 31-34.

53 Xu RH, Pelicano H, Zhou Y, Carew JS, Feng L, Bhalla KN et al. Inhibition of glycolysis in cancer cells: a novel strategy to overcome drug resistance associated with mitochondrial respiratory defect and hypoxia. Cancer Res 2005; 65: 613-621.

54 Geschwind JF, Ko YH, Torbenson MS, Magee C, Pedersen PL. Novel therapy for liver cancer: direct intraarterial injection of a potent inhibitor of ATP production. Cancer Res 2002; 62: 3909-3913.

55 Hulleman E, Kazemier KM, Holleman A, VanderWeele DJ, Rudin CM, Broekhuis $\mathrm{MJ}$ et al. Inhibition of glycolysis modulates prednisolone resistance in acute lymphoblastic leukemia cells. Blood 2009; 113: 2014-2021.

56 Del Bufalo D, Biroccio A, Soddu S, Laudonio N, D'Angelo C, Sacchi A et al. Lonidamine induces apoptosis in drug-resistant cells independently of the p53 gene. J Clin Invest 1996; 98: 1165-1173.

57 Li YC, Fung KP, Kwok TT, Lee CY, Suen YK, Kong SK. Mitochondria targeting drug lonidamine triggered apoptosis in doxorubicin-resistant HepG2 cells. Life Sci 2002; 71: 2729-2740.

58 Ravagnan L, Marzo I, Costantini P, Susin SA, Zamzami N, Petit PX et al. Lonidamine triggers apoptosis via a direct, Bcl-2-inhibited effect on the mitochondrial permeability transition pore. Oncogene 1999; 18: 2537-2546.

59 Brawer MK. Lonidamine: basic science and rationale for treatment of prostatic proliferative disorders. Rev Urol 2005; 7 (Suppl 7), S21-S26.

60 Ditonno P, Battaglia M, Selvaggio O, Garofalo L, Lorusso V, Selvaggi FP. Clinical evidence supporting the role of Lonidamine for the treatment of BPH. Rev Urol 2005; 7 (Suppl 7), S27-S33.

61 Gatenby RA, Gillies RJ. Glycolysis in cancer: a potential target for therapy. Int J Biochem Cell Biol 2007; 39: 1358-1366.

62 Lopez-Lazaro M. HIF-1: hypoxia-inducible factor or dysoxia-inducible factor? FASEB J 2006; 20: 828-832.

63 Erecinska M, Deas J, Silver IA. The effect of $\mathrm{pH}$ on glycolysis and phosphofructokinase activity in cultured cells and synaptosomes. J Neurochem 1995; 65: 2765-2772.

64 Trivedi B, Danforth WH. Effect of $\mathrm{pH}$ on the kinetics of frog muscle phosphofructokinase. J Biol Chem 1966; 241: 4110-4112.

65 Reshkin SJ, Bellizzi A, Caldeira S, Albarani V, Malanchi I, Poignee M et al. $\mathrm{Na}+/ \mathrm{H}+$ exchanger-dependent intracellular alkalinization is an early event in malignant transformation and plays an essential role in the development of subsequent transformation-associated phenotypes. FASEB J 2000; 14: 2185-2197.

66 Harguindey S, Orive G, Luis Pedraz J, Paradiso A, Reshkin SJ. The role of $\mathrm{pH}$ dynamics and the $\mathrm{Na}+/ \mathrm{H}+$ antiporter in the etiopathogenesis and treatment of cancer. Two faces of the same coin-one single nature. Biochim Biophys Acta 2005; 1756: 1-24.

67 Cardone RA, Casavola V, Reshkin SJ. The role of disturbed $\mathrm{pH}$ dynamics and the $\mathrm{Na}+/ \mathrm{H}+$ exchanger in metastasis. Nat Rev Cancer 2005; 5: $786-795$.

68 Evans DM, Sloan-Stakleff K, Arvan M, Guyton DP. Time and dose dependency of the suppression of pulmonary metastases of rat mammary cancer by amiloride. Clin Exp Metastasis 1998; 16: 353-357.

69 Sparks RL, Pool TB, Smith NK, Cameron IL. Effects of amiloride on tumor growth and intracellular element content of tumor cells in vivo. Cancer Res 1983; 43: 73-77.

70 Garber K. Energy deregulation: licensing tumors to grow. Science 2006; 312: 1158-1159.

71 Hatzivassiliou G, Zhao F, Bauer DE, Andreadis C, Shaw AN, Dhanak D et al. ATP citrate lyase inhibition can suppress tumor cell growth. Cancer Cell 2005; 8: 311-321.

72 Li JJ, Wang H, Tino JA, Robl JA, Herpin TF, Lawrence RM et al. 2-hydroxy$\mathrm{N}$-arylbenzenesulfonamides as ATP-citrate lyase inhibitors. Bioorg Med Chem Lett 2007; 17: 3208-3211.

73 Christofk HR, Vander Heiden MG, Harris MH, Ramanathan A, Gerszten RE, Wei $\mathrm{R}$ et al. The M2 splice isoform of pyruvate kinase is important for cancer metabolism and tumour growth. Nature 2008; 452: 230-233.

74 Mazurek S, Boschek CB, Hugo F, Eigenbrodt E. Pyruvate kinase type M2 and its role in tumor growth and spreading. Semin Cancer Biol 2005; 15: 300-308.

75 Vander Heiden MG, Cantley LC, Thompson CB. Understanding the Warburg effect: the metabolic requirements of cell proliferation. Science 2009; 324: 1029-1033.
76 Fang M, Shen Z, Huang S, Zhao L, Chen S, Mak TW et al. The ER UDPase ENTPD5 promotes protein N-glycosylation, the Warburg effect, and proliferation in the PTEN pathway. Cell 2010; 143: 711-724.

77 Marshall S, Bacote V, Traxinger RR. Discovery of a metabolic pathway mediating glucose-induced desensitization of the glucose transport system. Role of hexosamine biosynthesis in the induction of insulin resistance. J Biol Chem 1991; 266: 4706-4712.

78 Eigenbrodt E, Mazurek S, Grimm H Use of sugar phosphates, sugar phosphate analogs, amino acids and/or amino acid analogs for modulating the glucolysis-enzyme complex, the malate aspartate shuttle and/or the transaminases 2009. US20090163591.

79 Scheefers $\mathrm{H}$ Compounds for modulating the glycolysis enzyme complex and/or the transaminase complex 2009. US20090149415.

80 Wang JB, Erickson JW, Fuji R, Ramachandran S, Gao P, Dinavahi R et al. Targeting mitochondrial glutaminase activity inhibits oncogenic transformation. Cancer Cell 2010; 18: 207-219.

81 Fantin VR, St-Pierre J, Leder P. Attenuation of LDH-A expression uncovers a link between glycolysis, mitochondrial physiology, and tumor maintenance. Cancer Cell 2006; 9: 425-434.

82 Bui T, Thompson CB. Cancer's sweet tooth. Cancer Cell 2006; 9: 419-420.

83 Deck LM, Royer RE, Chamblee BB, Hernandez VM, Malone RR, Torres JE et al. Selective inhibitors of human lactate dehydrogenases and lactate dehydrogenase from the malarial parasite Plasmodium falciparum. J Med Chem 1998; 41: 3879-3887.

84 Zhou M, Zhao Y, Ding Y, Liu H, Liu Z, Fodstad 0 et al. Warburg effect in chemosensitivity: targeting lactate dehydrogenase- $A$ re-sensitizes taxolresistant cancer cells to taxol. Mol Cancer 2010; 9: 33

85 Stacpoole PW, Gilbert LR, Neiberger RE, Carney PR, Valenstein E, Theriaque DW et al. Evaluation of long-term treatment of children with congenital lactic acidosis with dichloroacetate. Pediatrics 2008; 121: e1223-e1228.

86 Stacpoole PW, Kurtz TL, Han Z, Langaee T. Role of dichloroacetate in the treatment of genetic mitochondrial diseases. Adv Drug Deliv Rev 2008; 60: $1478-1487$

87 Griguer CE, Oliva CR, Gillespie GY. Glucose metabolism heterogeneity in human and mouse malignant glioma cell lines. J Neurooncol 2005; 74: 123-133.

88 Carmeliet P, Dor Y, Herbert JM, Fukumura D, Brusselmans K, Dewerchin M et al. Role of HIF-1alpha in hypoxia-mediated apoptosis, cell proliferation and tumour angiogenesis. Nature 1998; 394: 485-490.

89 Bertout JA, Patel SA, Simon MC. The impact of 02 availability on human cancer. Nat Rev Cancer 2008; 8: 967-975.

90 Berridge MV, Herst PM, Tan AS. Metabolic flexibility and cell hierarchy in metastatic cancer. Mitochondrion 2010; 10: 584-588.

91 Chen JL, Lucas JE, Schroeder T, Mori S, Wu J, Nevins J et al. The genomic analysis of lactic acidosis and acidosis response in human cancers. PLoS Genet 2008; 4: e1000293.

92 Marusyk A, Polyak K. Tumor heterogeneity: causes and consequences. Biochim Biophys Acta 2010; 1805: 105-117.

$93 \mathrm{Wu} \mathrm{CH}, \mathrm{Ho}$ YS, Tsai CY, Wang YJ, Tseng H, Wei PL et al. In vitro and in vivo study of phloretin-induced apoptosis in human liver cancer cells involving inhibition of type II glucose transporter. Int J Cancer 2009; 124: 2210-2219.

94 Liu Y, Cao Y, Zhang W, Bergmeier S, Qian Y, Akbar H et al. A smallmolecule inhibitor of glucose transporter 1 downregulates glycolysis, induces cell-cycle arrest, and inhibits cancer cell growth in vitro and in vivo. Mol Cancer Ther 2012; 11: 1672-1682.

95 Le A, Cooper CR, Gouw AM, Dinavahi R, Maitra A, Deck LM et al. Inhibition of lactate dehydrogenase $A$ induces oxidative stress and inhibits tumor progression. Proc Natl Acad Sci USA 2010; 107: 2037-2042.

96 Vazquez-Martin A, Colomer R, Brunet J, Menendez JA. Pharmacological blockade of fatty acid synthase (FASN) reverses acquired autoresistance to trastuzumab (Herceptin) by transcriptionally inhibiting 'HER2 superexpression' occurring in high-dose trastuzumab-conditioned SKBR3/ Tzb100 breast cancer cells. Int J Oncol 2007; 31: 769-776.

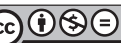

This work is licensed under a Creative Commons Attribution-NonCommercial-NoDerivs 3.0 Unported License. To view a copy of this license, visit http:// creativecommons.org/licenses/by-nc-nd/3.0/ 